Article

\title{
On the Decomposability of the Linear Combinations of Euler Polynomials with Odd Degrees
}

\author{
Ákos Pintér ${ }^{1, *}$ and Csaba Rakaczki ${ }^{2}$ \\ 1 Institute of Mathematics, MTA-DE Research Group “Equations, Functions and Curves”, Hungarian Academy of \\ Sciences and University of Debrecen, P. O. Box 12, H-4010 Debrecen, Hungary \\ 2 Institute of Mathematics, University of Miskolc, H-3515 Miskolc Campus, Hungary; matrcs@uni-miskolc.hu \\ * Correspondence: apinter@science.unideb.hu
}

Received: 2 April 2019; Accepted: 18 May 2019; Published: 31 May 2019

check for updates

\begin{abstract}
In the present paper we prove that under certain conditions the linear combination of two Euler polynomials with odd degrees $P_{n, m}(x)=E_{n}(x)+c E_{m}(x)$ is always indecomposable over $\mathbb{C}$, where $c$ denotes a rational number.
\end{abstract}

Keywords: Euler polynomials; higher degree equations

MSC: 11B68; 11B83; 11R09

\section{Introduction}

Let $\mathbb{K}$ be a field. If $f(x) \in \mathbb{K}[x]$ has degree at least 2, we say that $f(x)$ is decomposable over the field $\mathbb{K}$ if we can write $f(x)=f_{1}\left(f_{2}(x)\right)$ for some nonlinear polynomial $f_{1}(x), f_{2}(x) \in \mathbb{K}[x]$. Otherwise, we say that $f(x)$ is indecomposable over $\mathbb{K}$. Two decompositions $f(x)=f_{1}\left(f_{2}(x)\right)$ and $f(x)=F_{1}\left(F_{2}(x)\right)$ are said to be equivalent over the field $\mathbb{K}$, written $f_{1} \circ f_{2} \sim_{\mathbb{K}} F_{1} \circ F_{2}$, if there exists a linear polynomial $l(x) \in \mathbb{K}[x]$ such that

$$
f_{1}(x)=F_{1}(l(x)) \text { and } F_{2}(x)=l\left(f_{2}(x)\right) .
$$

For a given $f(x) \in \mathbb{K}[x]$ with degree at least 2, a complete decomposition of $f(x)$ over $\mathbb{K}$ is a decomposition $f=f_{1} \circ \cdots \circ f_{k}$, where the polynomials $f_{i} \in \mathbb{K}[x]$ are indecomposable over $\mathbb{K}$ for $i=1, \ldots, k$. A polynomial of degree greater than 1 always has a complete decomposition, but it does not need to be unique even up to equivalence.

Euler polynomials are defined by the following generating function

$$
\sum_{k=0}^{\infty} E_{k}(x) \frac{t^{k}}{k !}=\frac{2 e^{t x}}{\left(e^{t}+1\right)}
$$

These polynomials play a central role in various branches of mathematics; for example, in various approximation and expansion formulas in discrete mathematics and in number theory (see for instance [1,2]), in $p$-adic analyis (see [3], Chapter 2), in statistical physics as well as in semi-classical approximations to quantum probability distributions (see [4-7]).

There are several results connected to the decomposability of an infinite family of polynomials, see for instance [8-12]. Bilu, Brindza, Kirschenhofer, Pintér and Tichy [13] gave all the decompositions of Bernoulli polynomials. Kreso and Rakaczki [14] characterized the all possible decomposations of Euler 
polynomials with degree even, moreover they showed that every Euler polynomial with odd degree is indecomposable. It is harder to obtain similar results for the sum of polynomials. Pintér and Rakaczki [15] describe the complete decomposition of linear combinations of the form

$$
R_{n}(x)=B_{n}(x)+c B_{n-2}(x)
$$

of Bernoulli polynomials, where $c$ is an arbitrary rational number. Later, Pintér and Rakaczki in [16] proved that for all odd $n>1$ integer and for all rational number $c$ the polynomials $E_{n}(x)+c E_{n-2}(x)$ are indecomposable.

The main purpose of this paper is to prove that under certain conditions a linear combination with rational coefficients of two Euler polynomials with odd degrees is always indecomposable. We have

Theorem 1. Let $P_{n, m}(x)=E_{n}(x)+c E_{m}(x)$, where $c=A / B$ is an arbitrary rational number, where $B \neq 2^{a}$, $a \in \mathbb{N} \cup\{0\}, n, m$ are odd integers with $n>m>n / 3$. Then the polynomials $P_{n, m}(x)$ are indecomposable over $\mathbb{C}$.

\section{Auxiliary Results}

In the first lemma we collect some well known properties of the Euler polynomials which will be used in the sequel, sometimes without particular reference.

Lemma 1. (a) $E_{n}(x)=(-1)^{n} E_{n}(1-x)$;

(b) $E_{n}(x+1)+E_{n}(x)=2 x^{n}$;

(c) $E_{n}^{\prime}(x)=n E_{n-1}(x)$;

(d) $E_{2 n-1}(1 / 2)=E_{2 n}(0)=E_{2 n}(1)=0$ for $n \in \mathbb{N}$;

(e) $E_{n}(x)=\sum_{k=0}^{n}\left(\begin{array}{l}n \\ k\end{array}\right) E_{k}(0) x^{n-k}$;

Proof. See [2].

The following result is a general theorem from the theory of decomposability.

Lemma 2 (Kreso and Rakaczki [14]). Let $F(x) \in \mathbb{K}[x]$ be a monic polynomial such that $\operatorname{deg} F$ is not divisible by the characteristic of the field $\mathbb{K}$. Then for every nontrivial decomposition $F=F_{1} \circ F_{2}$ over any field extension $\mathbb{L}$ of $\mathbb{K}$, there exists a decomposition $F=\tilde{F}_{1} \circ \tilde{F}_{2}$ such that the following conditions are satisfied

- $\quad \tilde{F}_{1} \circ \tilde{F}_{2}$ and $F_{1} \circ F_{2}$ are equivalent over $\mathbb{L}$,

- $\quad \tilde{F}_{1}(x)$ and $\tilde{F}_{2}(x)$ are monic polynomials with coefficients in $\mathbb{K}$,

- $\quad \operatorname{coeff}\left(x^{\operatorname{deg} \tilde{F}_{1}-1}, \tilde{F}_{1}(x)\right)=0$.

Moreover, such decomposition $\tilde{F}_{1} \circ \tilde{F}_{2}$ is unique.

Lemma 3. Let $h(x) \in \mathbb{Q}[x]$ with $\operatorname{deg} h(x) \geq 4$. If $h(x)$ is decomposable over $\mathbb{Q}$ then we can write the polynomial $h(x)$ in the form $h(x)=\frac{u}{v} f(g(x))$, where $u$ and $v \neq 0$ are relative prime integers, $f(x)$ and $g(x) \in \mathbb{Z}[x]$ are primitive polynomials. Moreover, if $h(x)$ is a monic polynomial, then $u=1$.

Proof. Suppose that $h(x)=F(G(x))$, where $F(x), G(x) \in \mathbb{Q}[x]$. Let

$$
\begin{aligned}
& F(x)=b_{k} x^{k}+b_{k-1} x^{k-1}+\cdots+b_{1} x+b_{0}, \\
& G(x)=c_{t} x^{t}+c_{t-1} x^{t-1}+\cdots+c_{1} x+c_{0} .
\end{aligned}
$$


Every polynomial with rational coefficients can be written uniquely as a product of a rational number and a primitive polynomial. Hence, we can assume that

$$
G(x)=\frac{c}{d} g(x), \text { where } g(x) \text { is a primitive polynomial, } c, d \neq 0 \in \mathbb{Z}
$$

and so

$$
F(G(x))=b_{k}\left(\frac{c}{d}\right)^{k} g(x)^{k}+b_{k-1}\left(\frac{c}{d}\right)^{k-1} g(x)^{k-1}+\cdots+b_{1} \frac{c}{d} g(x)+b_{0} .
$$

The polynomial

$$
F_{1}(x)=b_{k}\left(\frac{c}{d}\right)^{k} x^{k}+b_{k-1}\left(\frac{c}{d}\right)^{k-1} x^{k-1}+\cdots+b_{1} \frac{c}{d} x+b_{0} \in \mathbb{Q}[x]
$$

can be written in the from $\frac{u}{v} f(x)$, where $f(x) \in \mathbb{Z}[x]$ is a primitive polynomial, $u>0, v \neq 0$ are relative prime integers. However, then we have

$$
h(x)=F(G(x))=F_{1}(g(x))=\frac{u}{v} f(g(x)) .
$$

If the polynomial $h(x)$ is monic, then comparing the leading coefficients in (1) one can deduce that $v=u f_{k} g_{t}^{k}$, where $f_{k}$ and $g_{t}$ denotes the leading coefficient of the polynomial $f(x)$ and $g(x)$, respectively. This means that $u$ divides $v$ that is $u=1$.

Let

$$
S^{+}=\{f(x) \in \mathbb{C}[x] \mid f(x)=f(1-x)\}
$$

and

$$
S^{-}=\{f(x) \in \mathbb{C}[x] \mid f(x)=-f(1-x)\} .
$$

From these definitions it is easy to see that $S^{+}$and $S^{-}$are subspaces in the vector space $\mathbb{C}[x]$.

Lemma 4. Let $P(x) \in \mathbb{Q}[x]$ be a monic polynomial. Assume that $P(x) \in S^{-}$and $P(x)=f(g(x))$, where $f(x)$, $g(x) \in \mathbb{Q}[x]$ and $\operatorname{deg}(f(x)), \operatorname{deg}(g(x))>1$. Then we can assume that $f(x), g(x)$ are monic, $g(x) \in S^{-}$and $f(x)=-f(-x)$.

Proof. See [16].

The following Lemma is a simple combination of Lemmas 3 and 4.

Lemma 5. Let $P(x) \in \mathbb{Q}[x]$ be a monic polynomial. Assume that $P(x) \in S^{-}$and $P(x)=F(G(x))$, where $F(x)$, $G(x) \in \mathbb{Q}[x]$ and $\operatorname{deg}(F(x))>1, \operatorname{deg}(G(x))>1$. Then we can assume that $P(x)=\frac{1}{v} f(g(x))$, where $v \neq 0$ is an integer, $f(x)$ and $g(x)$ are primitive polynomials, $g(x) \in S^{-}$and $f(x)=-f(-x)$.

Proof. From Lemma 4 we can assume that $G(x) \in S^{-}$and $F(x)=-F(-x)$. Using the proof of Lemma 3 and the fact that $S^{-}$is a subspace of $\mathbb{C}[x]$ we get the assertion of our Lemma.

Lemma 6. Let $g(x)=c_{t} x^{t}+c_{t-1} x^{t-1}+\cdots+c_{1} x+c_{0} \in S^{-}$. Then

$$
-2 c_{s}=\left(\begin{array}{c}
s+1 \\
s
\end{array}\right) c_{s+1}+\left(\begin{array}{c}
s+2 \\
s
\end{array}\right) c_{s+2}+\cdots+\left(\begin{array}{c}
t-1 \\
s
\end{array}\right) c_{t-1}+\left(\begin{array}{l}
t \\
s
\end{array}\right) c_{t}
$$

for even index $0 \leq s \leq t-1$. 
Proof. Since $g(x) \in S^{-}$we have that $-g(x)=g(1-x)$. Computing the coefficient of $x^{\mathcal{S}}$ on the both sides we obtain that

$$
-c_{s}=(-1)^{s}\left(c_{s}+\left(\begin{array}{c}
s+1 \\
s
\end{array}\right) c_{s+1}+\left(\begin{array}{c}
s+2 \\
s
\end{array}\right) c_{s+2}+\cdots+\left(\begin{array}{c}
t-1 \\
s
\end{array}\right) c_{t-1}+\left(\begin{array}{l}
t \\
s
\end{array}\right) c_{t}\right) .
$$

Lemma 7. Let

$$
\begin{gathered}
f(x)=b_{k} x^{k}+b_{k-2} x^{k-2}+b_{k-3} x^{k-3}+\cdots+b_{1} x+b_{0}, \\
g(x)=c_{t} x^{t}+c_{t-1} x^{t-1}+\cdots+c_{1} x+c_{0} \in \mathbb{Q}[x] .
\end{gathered}
$$

If $k, t \geq 2$ then the coefficient of the monomial $x^{k t-2}$ in the polynomial $f(g(x))$ is

$$
b_{k}\left(k c_{t}^{k-1} c_{t-2}+\left(\begin{array}{l}
k \\
2
\end{array}\right) c_{t}^{k-2} c_{t-1}^{2}\right) \text {. }
$$

Proof. It is easy to see that the monomial $x^{k t-2}$ occurs only in the term $b_{k} g(x)^{k}$. Expanding $g(x)^{k}$ we simply get the assertion.

\section{Proof of the Theorem}

Let $n, m$ be odd positive integers with $n-2>m>n / 3, B$ is an arbitrary integer which is not a power of two. The case of $m=n-2$ was treated in [16]. Suppose that $P_{n, m}(x)$ is decomposable over $\mathbb{C}$. From Lemmas 2 and 5 we can assume that $P_{n, m}(x)=\frac{1}{v} f(g(x))$, where $v \neq 0$ is an integer, $f(x)$, $g(x) \in \mathbb{Z}[x]$ are primitive polynomials and $g(x) \in S^{-}, f(x)=-f(-x)$. Let

$$
\begin{aligned}
& f(x)=b_{k} x^{k}+b_{k-2} x^{k-2}+b_{k-4} x^{k-4}+\cdots+b_{3} x^{3}+b_{1} x, \\
& g(x)=c_{t} x^{t}+c_{t-1} x^{t-1}+\cdots+c_{1} x+c_{0} .
\end{aligned}
$$

Using (b) of Lemma 1 one can deduce that

$$
\frac{1}{v} f(g(x+1))+\frac{1}{v} f(g(x))=P_{n, m}(x+1)+P_{n, m}(x)=2 x^{n}+2 c x^{m} .
$$

Since $P_{n, m}(x) \in S^{-}$thus $P_{n, m}(x+1)=-P_{n, m}(-x)$. From (3) we infer that the polynomial $g(x)-g(-x)$ divides the polynomial $P_{n, m}(x)-P_{n, m}(-x)=2 x^{n}+2 c x^{m}$, that is

$$
g(x)-g(-x)=d x^{s} h(x)
$$

where $d \in \mathbb{Q}, 0 \leq s \leq m$ and the polynomial $h(x)$ divides the polynomial $x^{n-m}+c$ in $\mathbb{Q}[x]$. We know that

$$
g(x)-g(-x)=2 c_{t} x^{t}+2 c_{t-2} x^{t-2}+\cdots+2 c_{3} x^{3}+2 c_{1} x .
$$

If the polynomial $h(x)$ is a constant polynomial then we have $t=s$ and so $c_{t-2}=0$. It follows from $P_{n, m}(x)=E_{n}(x)+c E_{m}(x)$ and (d), (e) of Lemma 1 that the coefficient of $x^{n-2}$ in $P_{n, m}(x)$ equals 0 . Applying now Lemma 7 we get that

$$
b_{k}\left(\begin{array}{l}
k \\
2
\end{array}\right) c_{t}^{k-2} c_{t-1}^{2}=0
$$

which is impossible since $-2 c_{t-1}=t c_{t}$ by Lemma 6 . 
In the case when $h(x)=x^{n-m}+c$ we get $s+n-m=t, d=2 c_{t}$ and $g(x)-g(-x)=2 c_{t} x^{s+n-m}+$ $2 c_{t} c x^{s}=2 c_{t} x^{t}+2 c_{t} c x^{t-(n-m)}$. Since by assumption $n-m>2$, we obtain again that $c_{t-2}=0$, which is not possible.

Next suppose that $1 \leq \operatorname{deg} h(x)<n-m$. In this case one can deduce that $s$ is odd and $h(x)=h(-x)$. Consider first when $\mathbf{1}<\mathbf{s}$. Then $c_{1}=c_{3}=\cdots=c_{s-2}=0$ and $c_{s} \neq 0$. Let $G(x)=g(x)-g(0)$ and $F(x)=f(x+g(0))$. Then $f(g(x))=F(G(x)), G(0)=0$ and

$$
G(x)-G(-x)=g(x)-g(-x)=2 c_{t} x^{t}+2 c_{t-2} x^{t-2}+\cdots+2 c_{s} x^{s} .
$$

Let

$$
F(x)=a_{k} x^{k}+a_{k-1} x^{k-1}+\cdots+a_{2} x^{2}+a_{1} x+a_{0} .
$$

Since $s<t \leq n / 3<m$ we have that $s+4 \leq m$.

Investigate the coefficients of $x^{s}$ and $x^{s+2}$ in

$$
v P_{n, m}(x)=F(G(x))=a_{k} G(x)^{k}+a_{k-1} G(x)^{k-1}+\cdots+a_{1} G(x)+a_{0} .
$$

Since $s+2<m$ in the polynomials $v P_{n . m}(x)=E_{n}(x)+c E_{m}(x)$ these coefficients are 0 . On the other hand, one can observe that $x^{\mathcal{S}}$ occurs only in the term $a_{1} G(x)$ and so $a_{1} c_{s}=0$. This means that $a_{1}=0$ and so

$$
v P_{n, m}(x)=F(G(x))=a_{k} G(x)^{k}+\cdots+a_{3} G(x)^{3}+a_{2} G(x)^{2}+a_{0} .
$$

Since $x^{s+2}$ appears only in the term $a_{2} G(x)^{2}$ thus $2 a_{2} c_{2} c_{s}=0$.

If $\mathbf{a}_{2}=\mathbf{0}$ we obtain from (7) that the coefficients of $x^{5}, x^{4}, x^{3}, x^{2}$ and $x$ in $F(G(x))$ are zero. This yields that $P_{n, m}^{(i)}(0)=0$ for $i=1, \ldots, 5$. Further, by Lemma 1

$$
\begin{gathered}
P_{n, m}^{(j)}(0)=P_{n, m}^{(j)}(1)=0, \text { if } j \text { is odd and } j \neq m, n ; \\
P_{n, m}^{(j)}\left(\frac{1}{2}\right)=0, \text { if } j \text { is even. }
\end{gathered}
$$

Applying the above, we can study the number of zeros of the polynomials $P_{n, m}^{(j)}(x)$ in the interval [0,1] for $j=1,2, \ldots, m+1$. In the following table we use only the Rolle's theorem.

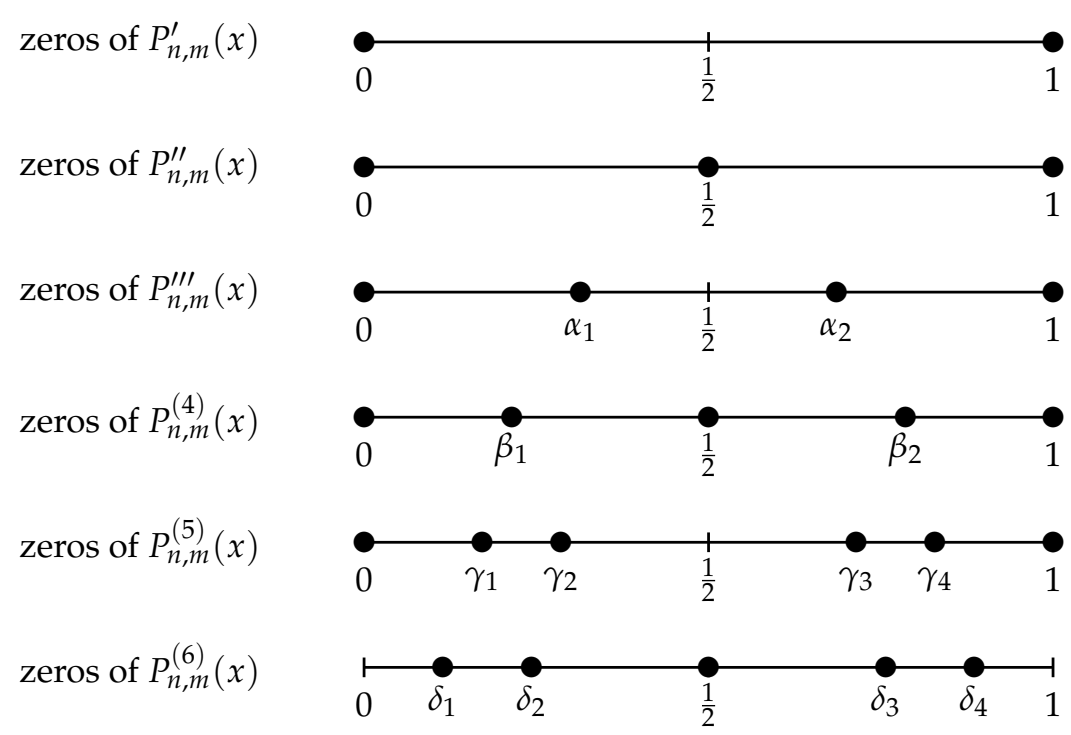


zeros of $P_{n, m}^{(7)}(x)$

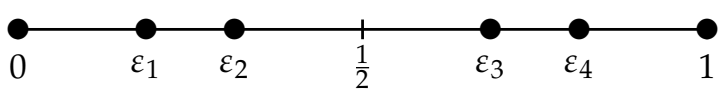

$$
P_{n, m}^{(m-1)}(x)
$$

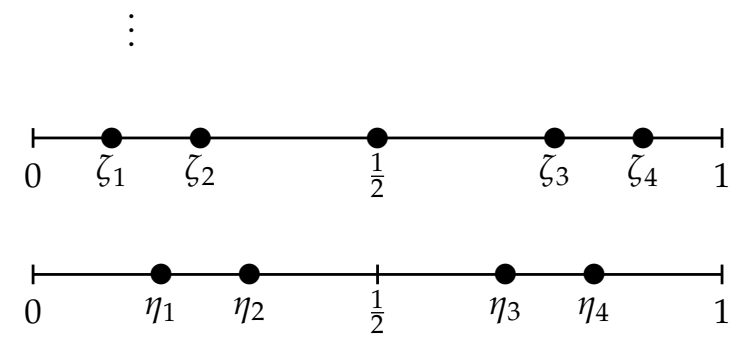

$P_{n, m}^{(m)}(x)$

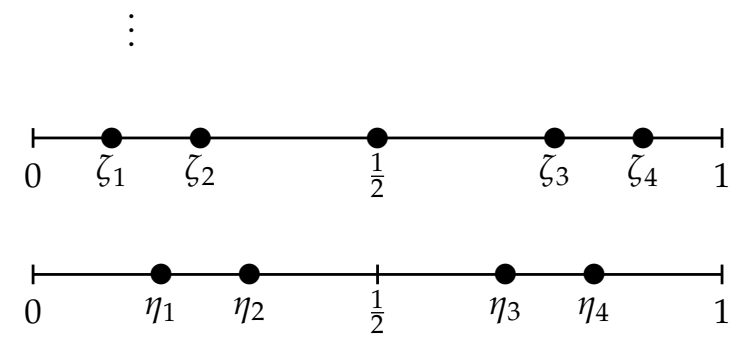

$P_{n, m}^{(m+1)}(x)$

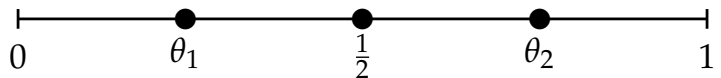

But

$$
P_{n, m}^{(m+1)}(x)=\frac{n !}{(n-m-1) !} E_{n-m-1}(x)
$$

whose the only zero in the interval $[0,1]$ is $1 / 2$. This contradiction gives that $a_{2} \neq 0$.

If $\mathbf{c}_{2}=\mathbf{0}$ then from $G(x)=c_{t} x^{t}+\cdots+c_{3} x^{3}$ and (7) one can deduce that

$$
P_{n, m}^{(j)}(0) \text { for } j=1,2,3,4,5 \text {. }
$$

The above argument that we used in the case $a_{2}=0$ shows that this impossible.

Finally, consider the case when $\mathbf{s}=\mathbf{1}$. Let $c=A / B$, where $A$ and $B \neq 0$ are relatively prime integers. From (4) we know that

$$
g(x)-g(-x)=2 c_{t} x^{t}+2 c_{t-2} x^{t-2}+\cdots+2 c_{3} x^{3}+2 c_{1} x=d x h(x),
$$

where the polynomial $h(x)$ is even and divides the polynomial $B x^{n-m}+A$ in $\mathbb{Q}[x]$. If we write $h(x)$ as a product of a rational number $a / b$ and a primitive polynomial $H(x)=h_{r} x^{r}+h_{r-2} x^{r-2}+\cdots+h_{2} x^{2}+h_{0}$ we have that

$$
B x^{n-m}+A=H(x) u(x),
$$

where $u(x)=u_{q} x^{q}+u_{q-2} x^{q-2}+\cdots+u_{2} x^{2}+u_{0}$ is a primitive polynomial. We obtain from (8) and (9) that

$$
\left(2 c_{t} x^{t}+2 c_{t-2} x^{t-2}+\cdots+2 c_{3} x^{3}+2 c_{1} x\right) u(x)=\frac{a}{b} d\left(B x^{n-m+1}+A x\right) .
$$

Let $c_{t}=w c_{t}^{\prime}, c_{t-2}=w c_{t-2}^{\prime}, \ldots, c_{3}=w c_{3}^{\prime}$ and $c_{1}=w c_{1}^{\prime}$, where $w$ denotes the greatest common divisor of the integers $c_{t}, c_{t-2}, \ldots, c_{3}, c_{1}$. Then

$$
2 w\left(c_{t}^{\prime} x^{t}+c_{t-2}^{\prime} x^{t-2}+\cdots+c_{3}^{\prime} x^{3}+c_{1}^{\prime} x\right) u(x)=\frac{a}{b} d\left(B x^{n-m+1}+A x\right),
$$

which yields that $2 w=(a / b) d$ and

$$
\left(c_{t}^{\prime} x^{t}+c_{t-2}^{\prime} x^{t-2}+\cdots+c_{1}^{\prime} x\right)\left(u_{q} x^{q}+u_{q-2} x^{q-2}+\cdots+u_{0}\right)=B x^{n-m+1}+A x .
$$


It follows from Lemma 6 that if $p$ is an odd prime which divides $w$ then $p$ divides $c_{t}, c_{t-1}, \ldots, c_{2}, c_{1}, c_{0}$ which is not possible since $g(x)$ is a primitive polynomial. Thus $w=2^{a}$ for some non-negative integer $a$. Now assume that $p$ is a prime which divides $c_{t}^{\prime}$ and $j \geq 1$ is the greatest odd index for which

$$
p \mid c_{t}^{\prime}, c_{t-2}^{\prime}, \ldots, c_{j+2}^{\prime} \text { and } p \nmid c_{j}^{\prime} \text {. }
$$

On the right hand side of (12) the coefficient of $x^{\alpha}$ equals 0 apart from when $\alpha=q+t=n-m+1$ or $\alpha=1$. Thus

$$
c_{j}^{\prime} u_{q}+c_{j+2}^{\prime} u_{q-2}+c_{j+4}^{\prime} u_{q-4}+\cdots=0
$$

which means that $p \mid u_{q}$.

Similarly,

$$
c_{j-2}^{\prime} u_{q}+c_{j}^{\prime} u_{q-2}+c_{j+2}^{\prime} u_{q-4}+\cdots=0
$$

from which we get that $p \mid u_{q-2}$. Continuing the process one can deduce that

$$
p \mid u_{q}, u_{q-2}, \ldots, u_{2}
$$

Further, if $j>1$ then

$$
c_{j}^{\prime} u_{0}+c_{j-2}^{\prime} u_{2}+\ldots=0
$$

and so $p \mid u_{0}$ contradicting that the polynomial $u(x)$ is a primitive polynomial. It follows from the above that $j$ must be 1 and so

$$
p \mid c_{t}^{\prime}, c_{t-2}^{\prime}, \ldots, c_{3}^{\prime}, u_{q}, u_{q-2}, \ldots, u_{2} \text { and } p \nmid c_{1}^{\prime}, u_{0} .
$$

If $p$ is an odd prime then from the above and Lemma 6 we have that

$$
p \mid c_{t}, c_{t-1}, c_{t-2} \ldots, c_{3}, c_{2} \text { and } p \nmid c_{1}, c_{0} .
$$

Now let $U(x)=c_{t} x^{t}+c_{t-1} x^{t-1}+\cdots+c_{2} x^{2}$ and $V(x)=c_{1} x+c_{0}$. Then $g(x)=U(x)+V(x)$ and for $j=0,1, \ldots, k$

$$
g(x)^{j}=\sum_{i=0}^{j}\left(\begin{array}{l}
j \\
i
\end{array}\right) U(x)^{j-i} V(x)^{i} \equiv V(x)^{j} \bmod (p) .
$$

We know that $m>n / 3 \geq k$ and so the coefficients of $x^{k}, x^{k-2}, \ldots, x^{3}, x$ are zeros in $P_{n, m}(x)$ and so in

$$
f(g(x))=b_{k} g(x)^{k}+b_{k-2} g(x)^{k-2}+\cdots+b_{3} g(x)^{3}+b_{1} g(x), \text { too. }
$$

Now one can infer from (16) and (17) that $0 \equiv b_{k} c_{1}^{k} \bmod (p)$ which yields $p \mid b_{k}$. Comparing coefficient of $x^{k-2}$ we have that $0 \equiv b_{k-2} c_{1}^{k-2} \bmod (p)$ from which we obtain $p \mid b_{k-2}$. Continuing the process it is easy to see that $p \mid b_{k-4}, \ldots, b_{3}, b_{1}$ which contradicts the fact that $f(x)$ is a primitive polynomial. This means that $c_{t}^{\prime}$ and $c_{t}$ must be powers of two.

Now suppose that $p$ is a prime with $p \mid u_{q}$ and $p \nmid c_{t}^{\prime}$. Using again that on the right hand side of (12) the coefficient of $x^{\alpha}$ equals 0 apart from when $\alpha=n-m+1$ or 1 . From $u_{q} c_{t-2}^{\prime}+u_{q-2} c_{t}^{\prime}=0$ we obtain $p$ divides $u_{q-2}$. From $u_{q} c_{t-4}^{\prime}+u_{q-2} c_{t-2}^{\prime}+u_{q-4} c_{t}^{\prime}=0$ we obtain $p$ divides $u_{q-4}$. It follows similarly that $p \mid u_{q-6}, \ldots, u_{2}$. Finally, from $c_{t}^{\prime} u_{0}+c_{t-2}^{\prime} u_{2}+\cdots=0$ we get that $p$ divides $u_{0}$ which contradicts that the polynomial $u(x)$ is a primitive polynomial. This means that $u_{q}$ must be a power of two. Since $B=c_{t}^{\prime} u_{q}$ this contradicts to our assumption that $B$ is not a power of two.

\section{Concluding Remarks}

It is a very hard problem to characterize the general decomposition of an infinite sequence of polynomials $f_{n}(x)$. The first theorem was proved for Bernoulli polynomials. For other results see our 
Introduction. A harder question is to describe the decomposition of the sum of two polynomials. There are only a few results in this direction, mainly for the rational linear combination of two Bernoulli and Euler polynomials in the form $B_{n}(x)+c B_{n-2}$ and $E_{n}(x)+c E_{n-2}(x)$, respectively. This paper contains the first theorem concerning the decomposition of the linear combination of two Euler polynomials $E_{m} x+c E_{n}(x)$ with "almost" independent parameters $m$ and $n$.

Author Contributions: All the authors contributed equally to the conception of the idea, implementing and analyzing the experimental results, and writing the manuscript.

Funding: This research Supported in part by the Hungarian Academy of Siences, and NKFIH/OTKA grant K128088.

Conflicts of Interest: The authors declare no conflict of interest.

\section{References}

1. Abramowitz, M.; Stegun, I. Handbook of Mathematical Functions with Formulas, Graphs and Mathematical Tables; Dover: New York, NY, USA, 1972.

2. Brillhart, J. On the Euler and Bernoulli polynomials. J. Reine Angew. Math. 1969, 234, 45-64.

3. Koblitz, N. p-adic Numbers, p-adic Analysis, and Zeta-Functions, 2nd ed.; Graduate Texts in Mathematics; Springer: New York, NY, USA, 1984.

4. Bagarello, F.; Trapani, C.; Triolo, S. Representable states on quasilocal quasi*-algebras. J. Math. Phys. 2011, 52, 013510. [CrossRef]

5. Ballentine, L.E.; McRae, S.M. Moment equations for probability distributions in classical and quantum mechanics. Phys. Rev. A 1998, 58, 1799-1809. [CrossRef]

6. Trapani, C.; Triolo, S. Representations of modules over a*-algebra and related seminorms. Stud. Math. 2008, 184, 133-148. [CrossRef]

7. Triolo, S. WQ* algebras of measurable operators. Indian J. Pure Appl. Math. 2012, 43, 601-617. [CrossRef]

8. Dujella, A.; Gusíc, I. Indecomposability of polynomials and related Diophantine equations. Q. J. Math. 2006, 57, 193-201. [CrossRef]

9. Dujella, A.; Gusíc, I. Decomposition of a recursive family of polynomials. Monatshefte für Mathematik 2007, 152, 97-104. [CrossRef]

10. Dujella, A.; Gusic, I.; Tichy, R.F. On the indecomposability of polynomials. Osterreich. Akad. Wiss. Math.-Natur. Kl. Sitzungsber. II 2005, 214, 81-88. [CrossRef]

11. Dujella, A.; Tichy, R.F. Diophantine equations for second-order recursive sequences of polynomials. Quart. J. Math. 2001, 52, 161-169. [CrossRef]

12. Kreso, D.; Tichy, R.F. Functional composition of polynomials: Indecomposability, Diophantine equations and lacunary polynomials. Graz. Math. Ber. 2015, 363, 143-170.

13. Bilu, Y.; Brindza, B.; Kirschenhofer, P.; Pintér, Á.; Tichy, R. Diophantine Equations and Bernoulli Polynomials. Compositio Math. 2002, 131, 173-188. [CrossRef]

14. Kreso, D.; Rakaczki, C. Diophantine equations with Euler polynomials. Acta Arith. 2013, 161, 267-281. [CrossRef]

15. Pintér, Á.; Rakaczki, C. On the decomposability of linear combinations of Bernoulli. Monatshefte Math. 2016, 180, 631-648. [CrossRef]

16. Pintér, Á.; Rakaczki, C. On the decomposability of the linear combinations of Euler polynomials. Miskolc Math. Notes 2017, 18, 407-415. [CrossRef]

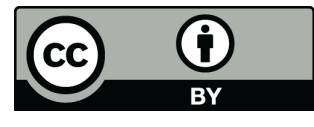

(C) 2019 by the authors. Licensee MDPI, Basel, Switzerland. This article is an open access article distributed under the terms and conditions of the Creative Commons Attribution (CC BY) license (http:/ / creativecommons.org/licenses/by/4.0/). 